EXTENDED REPORT

\title{
Non-invasive imaging in the diagnosis and management of Takayasu's arteritis
}

\author{
J Andrews, A Al-Nahhas, D J Pennell, M S Hossain, K A Davies, D O Haskard, J C Mason
}

Ann Rheum Dis 2004;63:995-1000. doi: 10.1136/ard.2003.015701

See end of article for authors' affiliations .....................

Correspondence to: Dr J Mason,

Cardiovascular Medicine Unit, Bywaters Centre, Imperial College London Hammersmith Hospital, Du Cane Road, London W12 ONN, UK; justin.mason@ imperial.ac.uk

Accepted 1 December 2003
Objectives: Takayasu's arteritis (TA) is a rare disease, in which early diagnosis and assessment of treatment efficacy remain a problem. Signs and symptoms may be non-specific and conventional blood tests unreliable, with vascular inflammation often persisting in the face of a normal acute phase response. The current "gold standard" investigation, $x$ ray angiography, is invasive and only identifies late, structural changes in vessels. Recently, non-invasive imaging methods have shown promise in the assessment of patients with TA.

Methods: The invasive and non-invasive imaging performed on all patients in the rheumatology department at the Hammersmith Hospital between May 1996 and May 2002 who fulfilled the ACR criteria for TA were reviewed. All patients were clinically active at diagnosis and were treated with high dose oral prednisolone and additional oral or intravenous immunosuppression.

Results: Non-invasive imaging methods ([ $\left.{ }^{18} \mathrm{~F}\right]$ fluorodeoxyglucose positron emission tomography $\left(\left[{ }^{18} \mathrm{~F}\right] \mathrm{FDG}-\mathrm{PET}\right)$ and magnetic resonance imaging (MRI)) provided important additional information about disease activity ([ $\left.\left.{ }^{18} \mathrm{~F}\right] \mathrm{FDG}-\mathrm{PET}\right)$ and progression of vessel wall thickening (MRI) when compared with $x$ ray angiography.

Conclusions: Non-invasive imaging methods provide useful additional information towards the diagnosis and management of TA. Such techniques may allow earlier diagnosis and more accurate assessment of response to treatment than conventional clinical assessment and/or angiography. Non-invasive imaging is likely to be useful in the management of other large vessel vasculitides.
$\mathrm{T}$ akayasu's arteritis (TA) is a rare granulomatous panarteritis of the large vessels, with an incidence of between 1.2 and 2.6 cases/million/year. ${ }^{1}$ It has a female preponderance and is most common in southeast Asia; its cause is unknown. In many ways, it is a difficult disease to manage. In the early inflammatory phase, non-specific systemic signs and symptoms such as arthralgias, night sweats, and weight loss often predominate, resulting in a significant diagnostic challenge. There are no specific laboratory abnormalities. Hoffman and Ahmed examined multiple serological tests, including tissue factor, von Willebrand factor, sICAM-1, sVCAM-1, and sE-selectin, and found that no test could distinguish between healthy volunteers and patients with active TA. ${ }^{2}$ In the later occlusive phase, it is now well recognised that vascular inflammation may persist, although the patient has achieved remission by clinical and biochemical criteria. In a review of surgical aortic biopsy specimens from patients with clinically inactive disease, $44 \%$ showed histological evidence of continuing vascular inflammation. ${ }^{3}$

The current "gold standard" investigation for the diagnosis and follow up of patients with TA is $x$ ray angiography. However, it has limitations inherent to an invasive procedure. Furthermore, it is unreliable in the diagnosis of TA during the early (potentially reversible) inflammatory phase as it only identifies late, fixed changes in lumen diameter without providing information on vessel wall (mural) inflammation or thickening. Recently, non-invasive methods such as magnetic resonance imaging (MRI; contrast enhanced and/ or angiography) and $\left[{ }^{18} \mathrm{~F}\right]$ fluorodeoxyglucose positron emission tomography ([ $\left.\left.{ }^{18} \mathrm{~F}\right] \mathrm{FDG}-\mathrm{PET}\right)$ scanning have been investigated for their ability to aid diagnosis and monitor disease activity in large vessel vasculitides. $\left[{ }^{18} \mathrm{~F}\right] \mathrm{FDG}$ is deoxyglucose labelled with fluorine-18, a positron emitting radionuclide that identifies areas of high glucose metabolic activity. High levels of glucose metabolism are typically seen not only in the myocardium and brain but also in other tissues if inflammation is present. ${ }^{4}$ MRI provides high resolution imaging of anatomical features, such as luminal changes and mural thickness, and also physiological information, such as the presence of mural enhancement or oedema, which are both features of vascular inflammation. ${ }^{5}$ Vessel wall thickening occurs early in the pre-stenotic inflammatory stage of the disease. Specific MR sequences can distinguish this finding and therefore may have an important role in the early detection of TA at a potentially reversible stage.

We investigated the potential role for non-invasive imaging in $(a)$ early diagnosis and $(b)$ assessment of continuing disease activity after immunosuppression in patients with TA.

\section{PATIENTS AND METHODS \\ Patients studied}

We retrospectively reviewed the management and outcome of patients newly diagnosed with TA at the Hammersmith Hospital between May 1996 and 2002. From our rheumatology database, we identified all patients who fulfilled the American College of Rheumatology 1990 criteria for the diagnosis of TA. ${ }^{6}$ Between May 1996 and 2002 six patients were newly diagnosed with TA according to these criteria (table 1). Their mean (SD) age at diagnosis was 29.5 (5.6) years. The mean (SD) length of follow up during this study was 26 (14.5) months. All six patients initially

Abbreviations: CRP, C reactive protein; ESR, erythrocyte sedimentation rate; $\left[{ }^{18} \mathrm{~F}\right] \mathrm{FDG}-\mathrm{PET},\left[{ }^{18} \mathrm{~F}\right]$ fluorodeoxyglucose positron emission

tomography; IA, intra-arterial; MRA, magnetic resonance angiography; MRI, magnetic resonance imaging; TA, Takayasu's arteritis 


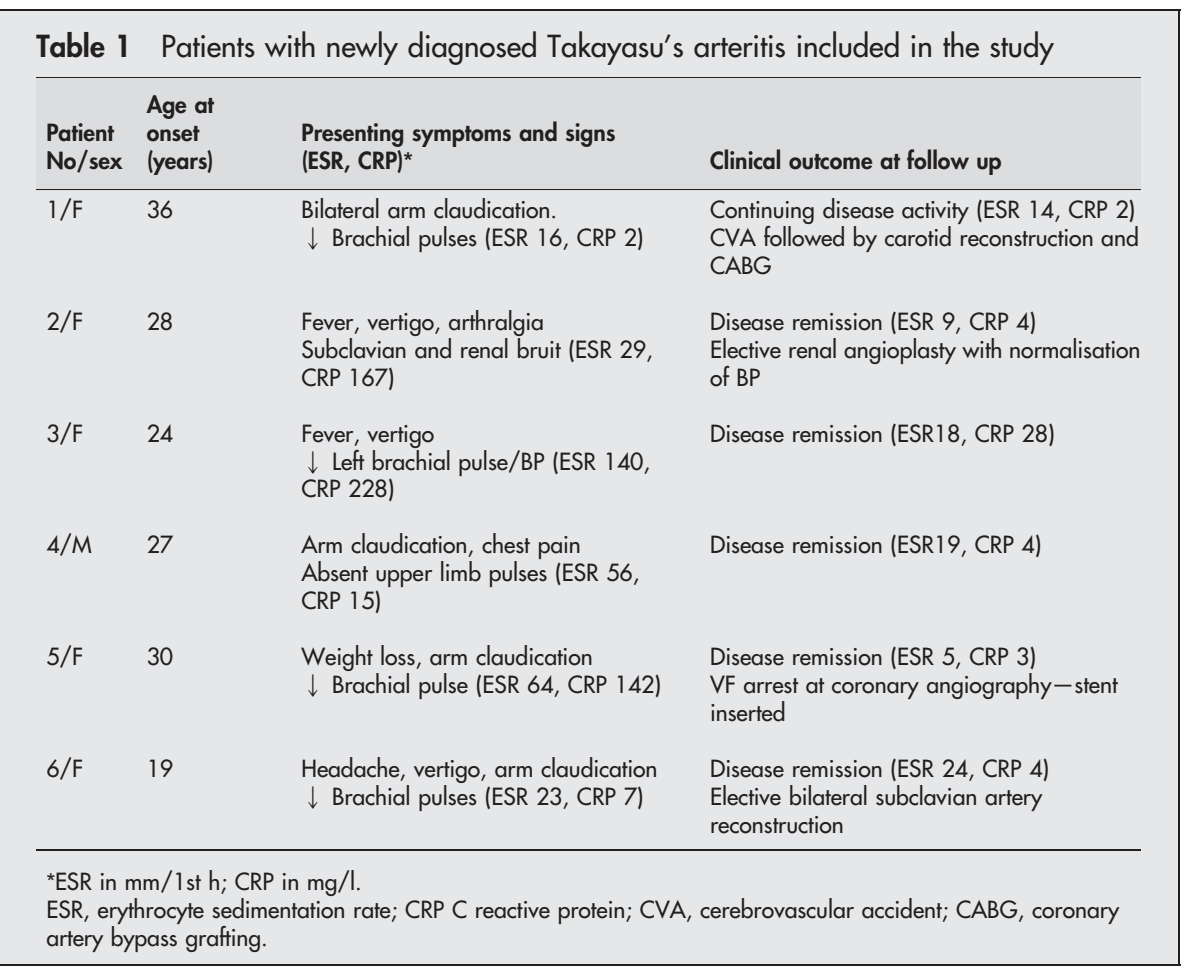

received oral prednisolone, four patients also received oral methotrexate (one patient was changed to mycophenolate because of side effects) and two patients received pulsed intravenous cyclophosphamide treatment. All patients fulfilled the criteria for active disease at diagnosis as defined by Kerr et al, ${ }^{3}$ which states that TA is active if there is new onset or worsening of two or more of the following features:

- Fever or arthralgia

- Raised erythrocyte sedimentation rate (ESR; >20 mm/ lst h)
- New claudication, bruit, or vascular pain

- New typical angiographic features.

\section{Imaging protocol}

During the period of review, it was our policy that conventional intra-arterial (IA) contrast angiography was always performed at diagnosis unless there was a contraindication. Patients also had either $\left[{ }^{18} \mathrm{~F}\right] \mathrm{FDG}$-PET or MRI (or both) performed at diagnosis and again during follow up. IA angiography was only performed at follow up if the noninvasive imaging results suggested continuing disease

Table 2 IA angiography, MRI, and $\left[{ }^{18} \mathrm{~F}\right] \mathrm{FDG}$-PET scan findings in patients with TA at diagnosis

\begin{tabular}{|c|c|c|c|c|c|c|c|c|}
\hline \multirow[b]{2}{*}{ Patient No } & \multirow[b]{2}{*}{ Imaging method } & \multicolumn{7}{|c|}{ Vascular area studied } \\
\hline & & $\begin{array}{l}\text { Ascending } \\
\text { aorta }\end{array}$ & $\begin{array}{l}\text { Aortic } \\
\text { arch }\end{array}$ & $\begin{array}{l}\text { Descending } \\
\text { aorta }\end{array}$ & $\begin{array}{l}\text { Abdominal } \\
\text { aorta }\end{array}$ & $\begin{array}{l}\text { Subclavian } \\
\text { arteries }\end{array}$ & $\begin{array}{l}\text { Carotid } \\
\text { arteries }\end{array}$ & $\begin{array}{l}\text { Vertebral } \\
\text { arteries }\end{array}$ \\
\hline 1 & $\begin{array}{l}\text { IA Angiogram } \\
\text { MRI/MRA } \\
{\left[{ }^{18} \text { F]FDG-PET }\right.}\end{array}$ & $\begin{array}{l}\text { Normal } \\
{ }^{*} \text { NP } \\
\text { Normal }\end{array}$ & $\begin{array}{l}\text { Normal } \\
{ }^{*} \text { NP } \\
\text { Normal }\end{array}$ & $\begin{array}{l}\text { Normal } \\
{ }^{*} \text { NP } \\
\text { Normal }\end{array}$ & $\begin{array}{l}\text { Stenotic } \\
{ }^{*} \text { NP } \\
\text { Normal }\end{array}$ & $\begin{array}{l}\text { Occluded } \\
{ }^{*} \mathrm{NP} \\
\uparrow \text { Uptake }\end{array}$ & $\begin{array}{l}\text { Occluded } \\
{ }^{*} \mathrm{NP} \\
\uparrow \text { Uptake }\end{array}$ & $\begin{array}{l}\text { Normal } \\
{ }^{*} \mathrm{NP} \\
\text { Normal }\end{array}$ \\
\hline 2 & $\begin{array}{l}\text { IA Angiogram } \\
\text { MRI/MRA } \\
{\left[{ }^{18} \mathrm{~F}\right] \mathrm{FDG}-\mathrm{PET}}\end{array}$ & $\begin{array}{l}\text { Normal } \\
\text { Normal } \\
\uparrow \text { Uptake }\end{array}$ & $\begin{array}{l}\text { Normal } \\
\text { Normal } \\
\uparrow \text { Uptake }\end{array}$ & $\begin{array}{l}\text { Normal } \\
\text { Normal } \\
\text { Normal }\end{array}$ & $\begin{array}{l}\text { Normal } \\
\text { Normal } \\
\text { Normal }\end{array}$ & $\begin{array}{l}\text { Stenotic } \\
\text { Stenotic } \\
\text { Normal }\end{array}$ & $\begin{array}{l}\text { Normal } \\
\text { Normal } \\
\uparrow \text { Uptake }\end{array}$ & $\begin{array}{l}\text { Stenotic } \\
\text { Normal } \\
\text { Normal }\end{array}$ \\
\hline 3 & $\begin{array}{l}\text { IA Angiogram } \\
\text { MRI/MRA } \\
{\left[{ }^{18} \text { F]FDG-PET }\right.}\end{array}$ & $\begin{array}{l}\text { Normal } \\
\text { Normal } \\
\uparrow \text { Uptake }\end{array}$ & $\begin{array}{l}\text { Normal } \\
\text { Normal } \\
\text { Normal }\end{array}$ & $\begin{array}{l}\text { Stenotic } \\
\text { Thickened } \\
\text { Normal }\end{array}$ & $\begin{array}{l}\text { Stenotic } \\
\text { Thickened } \\
\text { Normal }\end{array}$ & $\begin{array}{l}\text { Normal } \\
\text { Thickened } \\
\text { Normal }\end{array}$ & $\begin{array}{l}\text { Normal } \\
\text { Thickened } \\
\uparrow \text { Uptake }\end{array}$ & $\begin{array}{l}\text { Normal } \\
\text { Normal } \\
\text { Normal }\end{array}$ \\
\hline 4 & $\begin{array}{l}\text { IA Angiogram } \\
\text { MRI/MRA } \\
{\left[{ }^{18} \text { F]FDG-PET }\right.}\end{array}$ & $\begin{array}{l}{ }^{*} \mathrm{NP} \\
\text { Normal } \\
\text { Normal }\end{array}$ & $\begin{array}{l}{ }^{*} \text { NP } \\
\text { Normal } \\
\text { Normal }\end{array}$ & $\begin{array}{l}{ }^{*} \mathrm{NP} \\
\text { Normal } \\
\text { Normal }\end{array}$ & $\begin{array}{l}{ }^{*} \text { NP } \\
\text { Normal } \\
\text { Normal }\end{array}$ & $\begin{array}{l}{ }^{*} \mathrm{NP} \\
\text { Stenotic } \\
\text { Normal }\end{array}$ & $\begin{array}{l}\text { *NP } \\
\text { Occluded } \\
\text { Normal }\end{array}$ & $\begin{array}{l}{ }^{*} \text { NP } \\
\text { Normal } \\
\text { Normal }\end{array}$ \\
\hline 5 & $\begin{array}{l}\text { IA Angiogram } \\
\text { MRI/MRA } \\
{\left[{ }^{18} \mathrm{~F}\right] \mathrm{FDG}-\mathrm{PET}}\end{array}$ & $\begin{array}{l}\text { Stenotic } \\
\text { Thickened } \\
\uparrow \text { Uptake }\end{array}$ & $\begin{array}{l}\text { Normal } \\
\text { Thickened } \\
\uparrow \text { Uptake }\end{array}$ & $\begin{array}{l}\text { Normal } \\
\text { Thickened } \\
\uparrow \text { Uptake }\end{array}$ & $\begin{array}{l}\text { Normal } \\
\text { Normal } \\
\text { Normal }\end{array}$ & $\begin{array}{l}\text { Occluded } \\
\text { Occluded } \\
\text { Normal }\end{array}$ & $\begin{array}{l}\text { Normal } \\
\text { Thickened } \\
\uparrow \text { Uptake }\end{array}$ & $\begin{array}{l}\text { Normal } \\
\text { Thickened } \\
\text { Normal }\end{array}$ \\
\hline 6 & $\begin{array}{l}\text { IA Angiogram } \\
\text { MRI/MRA } \\
{\left[{ }^{18} \mathrm{~F}\right] \mathrm{FDG}-\mathrm{PET}}\end{array}$ & $\begin{array}{l}\text { Normal } \\
\text { Normal } \\
\text { Normal }\end{array}$ & $\begin{array}{l}\text { Normal } \\
\text { Normal } \\
\text { Normal }\end{array}$ & $\begin{array}{l}\text { Normal } \\
\text { Normal } \\
\text { Normal }\end{array}$ & $\begin{array}{l}\text { Normal } \\
\text { Normal } \\
\text { Normal }\end{array}$ & $\begin{array}{l}\text { Stenotic } \\
\text { Stenotic } \\
\uparrow \text { Uptake }\end{array}$ & $\begin{array}{l}\text { Stenotic } \\
\text { Stenotic } \\
\text { Normal }\end{array}$ & $\begin{array}{l}\text { Normal } \\
\text { Normal } \\
\text { Normal }\end{array}$ \\
\hline
\end{tabular}

*MRA, magnetic resonance angiography; NP, not performed. 
Table 3 IA angiography, MRI, and $\left[{ }^{18}\right.$ F]FDG-PET scan findings in patients with TA at follow up

\begin{tabular}{|c|c|c|c|c|c|c|c|c|}
\hline \multirow[b]{2}{*}{ Patient No } & \multirow[b]{2}{*}{ Imaging method } & \multicolumn{7}{|c|}{ Vascular area studied } \\
\hline & & $\begin{array}{l}\text { Ascending } \\
\text { aorta }\end{array}$ & $\begin{array}{l}\text { Aortic } \\
\text { arch }\end{array}$ & $\begin{array}{l}\text { Descending } \\
\text { aorta }\end{array}$ & $\begin{array}{l}\text { Abdominal } \\
\text { aorta }\end{array}$ & Subclavian arteries & Carotid arteries & Vertebral arteries \\
\hline 1 & $\begin{array}{l}\text { IA Angiogram } \\
\text { MRI/MRA } \\
{\left[{ }^{18} \mathrm{~F}\right] \text { FDG-PET }}\end{array}$ & $\begin{array}{l}\text { Normal } \\
{ }^{*} \mathrm{NP} \\
\text { Normal }\end{array}$ & $\begin{array}{l}\text { Normal } \\
{ }^{*} \mathrm{NP} \\
\text { Normal }\end{array}$ & $\begin{array}{l}\text { Normal } \\
{ }^{*} \mathrm{NP} \\
\text { Normal }\end{array}$ & $\begin{array}{l}\text { Stenotic } \\
{ }^{*} \mathrm{NP} \\
\text { Normal }\end{array}$ & $\begin{array}{l}\text { Occluded } \\
{ }^{*} \mathrm{NP} \\
\uparrow \text { Uptake }\end{array}$ & $\begin{array}{l}\text { Occluded } \\
{ }^{*} \mathrm{NP} \\
\uparrow \text { Uptake }\end{array}$ & $\begin{array}{l}\text { Normal } \\
{ }^{*} \mathrm{NP} \\
\text { Normal }\end{array}$ \\
\hline 2 & $\begin{array}{l}\text { IA Angiogram } \\
\text { MRI/MRA } \\
{\left[{ }^{18} \text { F]FDG-PET }\right.}\end{array}$ & $\begin{array}{l}\text { *NP } \\
\text { Normal } \\
\text { Normal }\end{array}$ & $\begin{array}{l}\text { *NP } \\
\text { Normal } \\
\text { Normal }\end{array}$ & $\begin{array}{l}\text { *NP } \\
\text { Normal } \\
\text { Normal }\end{array}$ & $\begin{array}{l}\text { *NP } \\
\text { Normal } \\
\text { Normal }\end{array}$ & $\begin{array}{l}{ }^{*} \mathrm{NP} \\
\text { Stenotic } \\
\text { Normal }\end{array}$ & $\begin{array}{l}\text { *NP } \\
\text { Normal } \\
\text { Normal }\end{array}$ & $\begin{array}{l}\text { *NP } \\
\text { Normal } \\
\text { Normal }\end{array}$ \\
\hline 3 & $\begin{array}{l}\text { IA Angiogram } \\
\text { MRI/MRA } \\
{ }^{18}{ }^{18} \text { F FDG-PET }\end{array}$ & $\begin{array}{l}\text { *NP } \\
\text { Normal } \\
\text { Normal }\end{array}$ & $\begin{array}{l}{ }^{*} \mathrm{NP} \\
\text { Normal } \\
\text { Normal }\end{array}$ & $\begin{array}{l}\text { *NP } \\
\text { Normal } \\
\text { Normal }\end{array}$ & $\begin{array}{l}\text { *NP } \\
\text { Normal } \\
\text { Normal }\end{array}$ & $\begin{array}{l}{ }^{* N P} \\
\text { Normal } \\
\text { Normal }\end{array}$ & $\begin{array}{l}{ }^{* N P} \\
\text { Normal } \\
\text { Normal }\end{array}$ & $\begin{array}{l}\text { *NP } \\
\text { Thickened } \\
\text { Normal }\end{array}$ \\
\hline 4 & $\begin{array}{l}\text { IA Angiogram } \\
\text { MRI/MRA } \\
{\left[{ }^{18} \text { F]FDG-PET }\right.}\end{array}$ & $\begin{array}{l}\text { *NP } \\
\text { Normal } \\
\text { Normal }\end{array}$ & $\begin{array}{l}{ }^{* N P} \\
\text { Normal } \\
\text { Normal }\end{array}$ & $\begin{array}{l}\text { *NP } \\
\text { Thickened } \\
\text { Normal }\end{array}$ & $\begin{array}{l}{ }^{*} \mathrm{NP} \\
\text { Normal } \\
\text { Normal }\end{array}$ & $\begin{array}{l}{ }^{*} \mathrm{NP} \\
\text { Stenotic } \\
\text { Normal }\end{array}$ & $\begin{array}{l}{ }^{*} \mathrm{NP} \\
\text { Occluded } \\
\text { Normal }\end{array}$ & $\begin{array}{l}\text { *NP } \\
\text { Normal } \\
\text { Normal }\end{array}$ \\
\hline 5 & $\begin{array}{l}\text { IA Angiogram } \\
\text { MRI/MRA } \\
{\left[{ }^{18} \mathrm{~F}\right] F D G-P E T}\end{array}$ & $\begin{array}{l}{ }^{* N P} \\
\text { Thickened } \\
\downarrow \text { Uptake }\end{array}$ & $\begin{array}{l}\text { *NP } \\
\text { Thickened } \\
\text { Normal }\end{array}$ & $\begin{array}{l}\text { *NP } \\
\text { Thickened } \\
\text { Normal }\end{array}$ & $\begin{array}{l}\text { *NP } \\
\text { Normal } \\
\text { Normal }\end{array}$ & $\begin{array}{l}\text { *NP } \\
\text { Occluded } \\
\text { Normal }\end{array}$ & $\begin{array}{l}\text { *NP } \\
\text { Thickened } \\
\downarrow \text { Uptake }\end{array}$ & $\begin{array}{l}\text { *NP } \\
\text { Thickened } \\
\text { Normal }\end{array}$ \\
\hline 6 & $\begin{array}{l}\text { IA Angiogram } \\
\text { MRI/MRA } \\
{\left[{ }^{18} \text { F]FDG-PET }\right.}\end{array}$ & $\begin{array}{l}\text { *NP } \\
\text { Normal } \\
\text { Normal }\end{array}$ & $\begin{array}{l}{ }^{*} \mathrm{NP} \\
\text { Normal } \\
\text { Normal }\end{array}$ & $\begin{array}{l}{ }^{*} \mathrm{NP} \\
\text { Normal } \\
\text { Normal }\end{array}$ & $\begin{array}{l}{ }^{*} \mathrm{NP} \\
\text { Normal } \\
\text { Normal }\end{array}$ & $\begin{array}{l}{ }^{*} \mathrm{NP} \\
\text { Stenotic } \\
\text { Normal }\end{array}$ & $\begin{array}{l}{ }^{*} \mathrm{NP} \\
\text { Stenotic } \\
\text { Normal }\end{array}$ & $\begin{array}{l}{ }^{*} \mathrm{NP} \\
\text { Normal } \\
\text { Normal }\end{array}$ \\
\hline
\end{tabular}

activity. IA angiography was performed using biplane aortography via the femoral artery route.

\section{$\left[{ }^{18} \mathrm{~F}\right]$ FDG-PET imaging}

After a fasting period of 4 hours before scanning, doses of between 185 and $259 \mathrm{MBq}(5-7 \mathrm{mCi}) \quad\left[{ }^{18} \mathrm{~F}\right] \mathrm{FDG}$ were injected intravenously based on patient weight and aimed at keeping the dead time to $<25 \%$. This gave an effective dose of $7 \mathrm{mSv}$ for the maximum doses of $259 \mathrm{MBq}$. Patients were rested for 90 minutes in a quiet room before scanning.

Whole body images from the base of the brain to midfemur were obtained using the dedicated Siemens ECAT ART positron emission tomograph. A transmission scan was performed using a ${ }^{137} \mathrm{Cs}$ source. Scan time, which was dependent on the height of the patient, averaged 90 minutes. Iterative reconstruction was performed on the emission scan and filtered back projection on the transmission scan. A simultaneous three dimensional acquisition was performed at the same time as the three dimensional image reconstruction and processing occurred. Results were reported as ratios to normalise any factor that might affect uptake. The maximum standardised uptake value in a rectangular region of interest over the large vessel (carotid, subclavian, or aortic vessel) which had maximal uptake was divided by the maximum standardised uptake value in a rectangular area over the lung at the same slice of the coronal PET. Ratios were then compared with those of normal controls (subjects referred for $\left[{ }^{18} \mathrm{~F}\right] \mathrm{FDG}$-PET imaging for oncological reasons without a history of lung cancer). Uptake of $\left[{ }^{18} \mathrm{~F}\right] \mathrm{FDG}$ was reported semiquantitatively: $0=$ no increased uptake, $1=$ mildly increased uptake, 2 =moderately increased uptake, and 3 = greatly increased uptake (table 4 ).

\section{MRI}

MRI was performed using the 1.5 T Sonata scanner (Siemens Medical Systems, Erlangen, Germany), with ECG-gated sequences. Multiple spin echo and gradient echo cines were acquired in the long and short axis of the aorta. MR angiography (MRA) was also performed using gadopentetate dimeglumine (Magnevist) $0.2 \mathrm{mmol} / \mathrm{kg}$. This protocol was specifically devised to look for mural thickening and arterial stenosis.

\section{Statistical analysis}

Means were compared using the Student's $t$ test using GraphPad Prism 4 (GraphPad Software Inc, San Diego, CA). Values of $\mathrm{p}<0.05$ were considered significant.

\section{RESULTS}

\section{Clinical findings}

The mean (SD) C reactive protein (CRP) and ESR at diagnosis were $96(94) \mathrm{mg} / \mathrm{ml}$ and 55 (44) $\mathrm{mm} / \mathrm{lst} \mathrm{h}$, respectively,

Table 4 Summary of imaging and clinical findings before and after treatment

\begin{tabular}{|c|c|c|c|c|c|c|c|c|}
\hline \multirow{2}{*}{$\begin{array}{l}\text { Patient } \\
\text { No }\end{array}$} & \multicolumn{3}{|c|}{ Initial imaging findings } & \multirow{2}{*}{$\begin{array}{l}\text { Disease activity } \\
\text { at diagnosis* }\end{array}$} & \multicolumn{3}{|c|}{ Follow up imaging findings } & \multirow{2}{*}{$\begin{array}{l}\text { Disease activity } \\
\text { at follow up* }\end{array}$} \\
\hline & Angiogram & {$\left[{ }^{18}\right]$ FDG-PET $\dagger$} & MRI/MRA & & Angiogram & {$\left[{ }^{18}\right]$ FDG-PET $\dagger$} & MRI/MRA & \\
\hline 1 & Occluded vessels & $\uparrow$ Uptake (2) & ${ }^{*} \mathrm{NP}$ & Active & Further occlusion & $\uparrow$ Uptake (2) & ${ }^{*} \mathrm{NP}$ & Active \\
\hline 2 & Stenotic vessels & $\uparrow$ Uptake (3) & Stenotic vessel & Active & ${ }^{*} \mathrm{NP}$ & Normal (0) & No progression & Remission \\
\hline 3 & Stenotic vessels & $\uparrow$ Uptake (2) & Thickened vessels & Active & ${ }^{*} \mathrm{NP}$ & Normal (0) & Resolution wall thickening & Remission \\
\hline 4 & ${ }^{*} \mathrm{NP}$ & Normal (0) & Occluded vessels & Active & ${ }^{*} \mathrm{NP}$ & Normal (0) & No progression & Remission \\
\hline 5 & Occluded vessels & $\uparrow$ Uptake (3) & Occluded vessels & Active & ${ }^{*} \mathrm{NP}$ & $\downarrow$ Uptake (1) & No progression & Remission \\
\hline 6 & Stenotic vessels & $\uparrow$ Uptake (1) & Stenotic vessels & Active & ${ }^{*} \mathrm{NP}$ & Normal (0) & No progression & Remission \\
\hline
\end{tabular}




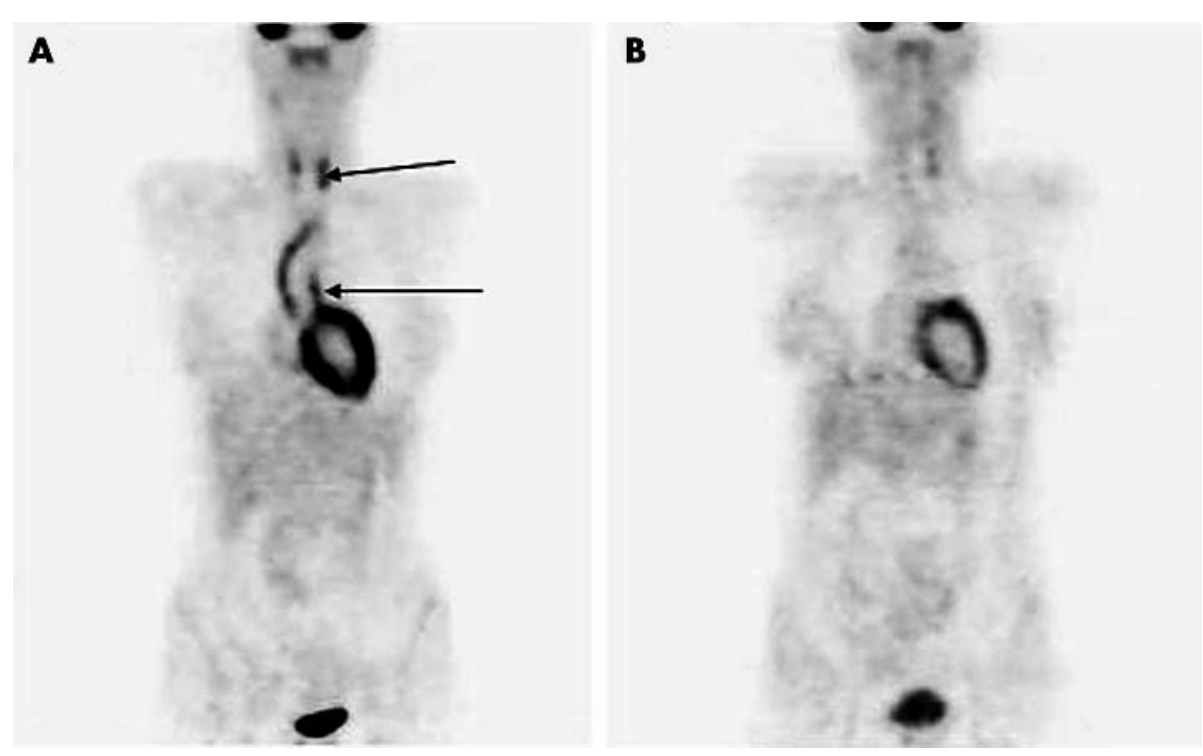

Figure 1 (A) $\left[{ }^{18}\right.$ F]FDG-PET scan of patient 5 with active TA at diagnosis. Note the markedly abnormal uptake of $\left[{ }^{18} \mathrm{~F}\right] \mathrm{FDG}$ in the aortic arch and carotid arteries (arrows). (B) [ $\left.{ }^{18} \mathrm{~F}\right] \mathrm{FDG}$-PET scan of the same patient in remission after treatment with prednisolone and intravenous cyclophosphamide. Note almost complete resolution of abnormal $\left[{ }^{18} \mathrm{~F}\right] \mathrm{FDG}$ uptake in these areas.

falling to a mean of $8(10) \mathrm{mg} / \mathrm{ml}$ and $11(6) \mathrm{mm} / \mathrm{lst} \mathrm{h}$ at follow up (see table 1). When the criteria developed by Kerr et $a l^{3}$ were used, five of the six patients achieved clinical disease remission during follow up. The one patient who failed to achieve clinical disease remission according to these criteria (patient 1) had a cerebrovascular accident and also underwent successful coronary artery bypass grafting and complex great vessel and aortic root reconstruction during follow up. For the five patients who achieved clinical disease remission, no cardiovascular events were recorded. However, one patient underwent successful renal angioplasty for renal artery stenosis (patient 2) and one patient had successful reconstructive surgery of the great vessels during the follow up (patient 6).

\section{Imaging findings}

Five of the six patients had conventional IA angiography at baseline. One patient was found to have a perfusion defect on myocardial thallium scanning consistent with a left main stem coronary artery occlusion; therefore invasive angiography was deemed too high risk to be performed (patient 4). Only the one patient who did not achieve clinical disease remission (patient 1) had repeat IA angiography at follow up. All six patients had an $\left[{ }^{18} \mathrm{~F}\right] \mathrm{FDG}$-PET scan at baseline and a repeat study during the follow up. Five patients had MRI performed at baseline and also during the follow up (patients $2,3,4,5$, and 6). Table 2 shows the results of the IA angiography, MRI, and $\left[{ }^{18} \mathrm{~F}\right]$ FDG-PET investigations at baseline, and table 3 the results at follow up.
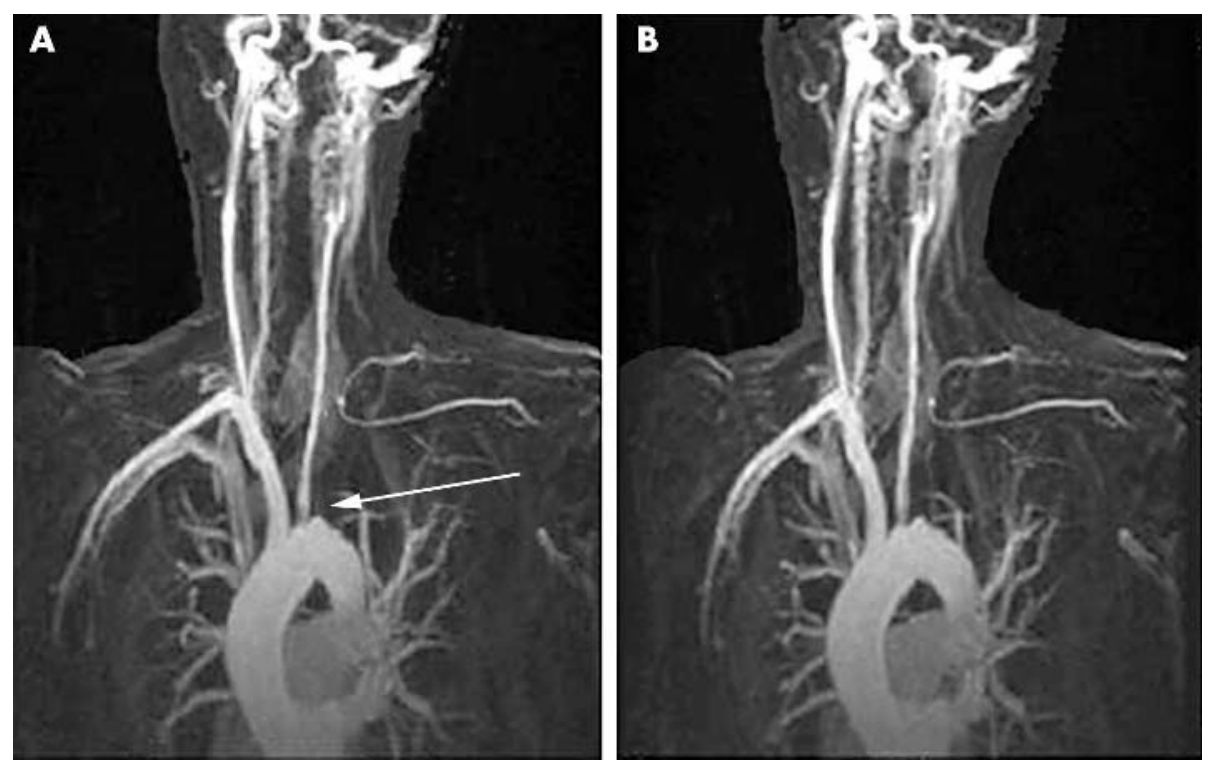

Figure 2 (A) Magnetic resonance angiography (MRA) image from patient 5 with active TA at diagnosis. There is complete occlusion of the left subclavian artery at its origin (arrow) with numerous collaterals evident and an ostial stenosis of the left common carotid artery. (B) MRA image from the same patient in remission. No significant progression of the lesions found on the baseline MRA is seen. 


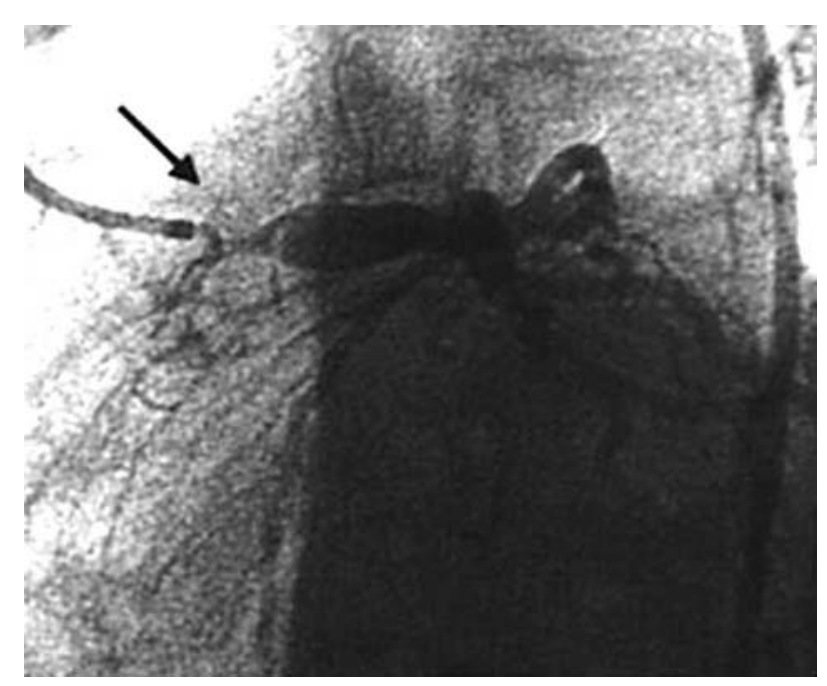

Figure 3 Coronary angiography image from patient number 5 with active TA at diagnosis. A tight stenosis can be seen at the origin of the left main stem coronary artery (arrow). Shortly after this image was recorded, she had a VF cardiac arrest and the left main stem was found to have occluded. After resuscitation, the left main stem was rapidly reopened with the insertion of a coronary stent without further complication.

Table 4 summarises the imaging findings of our six patients with TA at diagnosis and during follow up with their clinical disease activity assessments (using the criteria devised by Kerr et $a l^{3}$ ) during this period. In all six patients, invasive or non-invasive imaging methods performed at baseline confirmed anatomical changes consistent with a diagnosis of TA. All six patients were found to be clinically active at first presentation. After immunosuppressive treatment, five of the six patients achieved clinical disease remission, which was associated with a significant fall in the level of CRP $(p<0.04)$ and disease activity $(p=0.02)$. The non-invasive imaging procedures performed on these five patients corresponded with their clinical state, as all $\left[{ }^{18} \mathrm{~F}\right]$ FDG-PET scans performed at follow up showed significantly reduced $\left[{ }^{18} \mathrm{~F}\right] \mathrm{FDG}$ uptake in their vasculature $(\mathrm{p}<0.04)$. Furthermore, all MRI procedures performed at follow up confirmed that there had not been any anatomical disease progression, and in one case (patient 3) showed that some resolution of vessel wall thickening had occurred after treatment. The $\left[{ }^{18} \mathrm{~F}\right]$ FDG-PET scan of the one patient who did not achieve clinical disease remission after treatment (patient 1) continued to show abnormal uptake of $\left[{ }^{18} \mathrm{~F}\right] \mathrm{FDG}$ in the large vessels. Having initially achieved clinical remission at follow up, patient 2 developed a further flare of her clinical disease activity, with an episode of raised inflammatory markers, episodic visual loss, and angiodynia in the area of the right subclavian artery. A further $\left[{ }^{18} \mathrm{~F}\right] \mathrm{FDG}$ PET scan was performed and confirmed a new increased uptake of the $\left[{ }^{18} \mathrm{~F}\right] \mathrm{FDG}$ tracer in the root of aorta and right subclavian artery. After further immunosuppression, she is once again clinically inactive. We are awaiting further $\left[{ }^{18} \mathrm{~F}\right]$ FDG-PET imaging to confirm that that this clinical remission is reflected by a reduction in her vascular inflammation.

Figures $1 \mathrm{~A}$ and $\mathrm{B}$ show the $\left[{ }^{18} \mathrm{~F}\right] \mathrm{FDG}$-PET scans of patient 5 taken at diagnosis and after treatment, respectively. The markedly abnormal uptake of $\left[{ }^{18} \mathrm{~F}\right] \mathrm{FDG}$ in the aortic arch and carotid arteries can be seen in the pretreatment scan (fig 1A). The follow up scan (fig 1B) was taken when the patient was clinically in remission after treatment with prednisolone and intravenous cyclophosphamide (ESR
$5 \mathrm{~mm} / \mathrm{lst} \mathrm{h}, \mathrm{CRP} 3 \mathrm{mg} / \mathrm{ml}$ ). Note the almost complete resolution of abnormal $\left[{ }^{18} \mathrm{~F}\right] \mathrm{FDG}$ uptake in the previously inflamed areas. However, there is some residual uptake of $\left[{ }^{18} \mathrm{~F}\right]$ FDG in the carotid arteries suggestive of continuing low grade inflammation despite the inflammatory markers having normalised. Figures $2 \mathrm{~A}$ and B show MR images from patient 5 at diagnosis and follow up, respectively. In fig $2 \mathrm{~A}$ complete occlusion of the left subclavian artery with numerous collaterals is evident. There is an ostial stenosis of the left common carotid artery. No significant progression of these lesions is seen on the follow up image (fig 2B). Figure 3 shows an image from the coronary angiogram performed at diagnosis in the same patient.

\section{DISCUSSION}

Until now, IA angiography has been the main imaging method used in the diagnosis and management of TA. In addition to the fact that this method is invasive and necessitates a dose of radiographic dye that may compromise renal function, its use is principally limited to the detection of changes in lumen diameter, which occur at a relatively late stage of the disease. Our results suggest that new noninvasive imaging methods such as $\left[{ }^{18} \mathrm{~F}\right] \mathrm{FDG}-\mathrm{PET}$ and MRI can provide important additional information in TA about the presence of vascular inflammation and/or wall thickening when compared with conventional angiography. Early recognition of vascular inflammation or wall thickening is an important therapeutic advance, as it may allow TA to be diagnosed in the early pre-stenotic phase of the disease when it is potentially reversible and when patients' symptoms are often non-specific. In addition, it will also allow the response to treatment to be closely monitored during follow up.

$\left[{ }^{18} \mathrm{~F}\right]$ FDG-PET provides a useful measure of vascular inflammation in TA and with a single scan allows identification of all the vessels affected. In our study, low grade abnormal uptake of $\left[{ }^{18} \mathrm{~F}\right] \mathrm{FDG}$ remained detectable on the follow up scan of one patient (patient 5) when she was clinically in remission and the inflammatory markers had normalised, suggesting that $\left[{ }^{18} \mathrm{~F}\right]$ FDG-PET may be a more sensitive measure of continuing vascular inflammation than conventional clinical methods. Moreover, this observation concurs with the report of histological evidence of persistent vascular inflammation in biopsy specimens obtained from patients in apparent clinical remission. ${ }^{3}$

MRI also proved helpful in the analysis of disease distribution, in particular the presence or absence of wall thickening without frank stenosis. In one patient (patient 3), we could demonstrate on MRI reversible anatomical changes in the vessel wall after immunosuppressive treatment. Therefore, in addition to facilitating early diagnosis, these modalities will be useful not only in the assessment of the effect of drug treatment but also when contemplating interventional or surgical procedures, which are best performed during disease remission.

It should be noted, however, that overestimation of the degree of stenosis by up to $15 \%$ has been reported in some MRA studies. ${ }^{7}$ Furthermore, we did not find the presence or absence of gadolinium enhancement of the vessel wall on MRI a reliable guide to disease activity. Our findings are consistent with another recent study, which also found that assessing the presence or absence of vessel wall oedema in MR images was an inconsistent guide to the subsequent anatomical changes found by conventional angiography. ${ }^{8}$ In our experience, the presence or absence of $\left[{ }^{18} \mathrm{~F}\right]$ FDG uptake in the vessel wall on PET imaging proved to be a much more reliable guide to disease activity.

Blockmans et al have provided a substantial amount of data demonstrating the usefulness of $\left[{ }^{18} \mathrm{~F}\right] \mathrm{FDG}$-PET imaging in the early diagnosis of aortitis-in particular, giant cell 
arteritis and polymyalgia rheumatica. ${ }^{910}$ Another group, Meller et al, have also reported on the diagnostic value of $\left[{ }^{18} \mathrm{~F}\right]$ FDG-PET and MRI in the diagnosis of patients with aortitis. ${ }^{11}{ }^{12}$ However, our study is the first to report their use not only in the diagnosis but also in the follow up and assessment of response to treatment in a series of patients specifically with TA.

In summary, we have found that non-invasive imaging methods such as $\left[{ }^{18} \mathrm{~F}\right]$ FDG-PET scanning and MRI provided us with useful information for the early diagnosis and management of TA. Such techniques may allow earlier diagnosis and more accurate assessment of response to treatment than conventional clinical assessment and/or IA angiography. We suggest that further larger clinical studies are performed to compare the usefulness of invasive versus non-invasive imaging methods in the diagnosis and follow up of patients with TA. In the future, $\left[{ }^{18} \mathrm{~F}\right]$ FDG-PET and MRI may become the standard investigations performed when the diagnosis of TA is being considered and may ultimately replace the need for IA angiography in most patients.

\section{Authors' affiliations}

J Andrews, D O Haskard, J C Mason, Rheumatology Section, The Eric Bywaters Centre, Imperial College London, Hammersmith Hospital, Du Cane Road, London W12 ONN, UK

A Al-Nahhas, M S Hossain, Department of Nuclear Medicine, Faculty of Medicine, Imperial College London, Hammersmith Hospital, Du Cane Road, London W12 ONN, UK

D J Pennell, Department of Cardiovascular MR, National Heart and Lung Institute, Faculty of Medicine, Imperial College London, Royal Brompton Hospital, Sydney Street, London SW3 6NP, UK
K A Davies, Brighton and Sussex Medical School, University of Sussex, Brighton BN1 9PX, UK

\section{REFERENCES}

1 Hall S, Barr W, Lie JT, Stanson AW, Kazmier FJ, Hunder GG. Takayasu's arteritis: a study of 32 North American patients. Medicine (Baltimore) 1985;64:89-99.

2 Hoffman GS, Ahmed AE. Surrogate markers of disease activity in patients with Takayasu arteritis. Int J Cardiol 1998;66(suppl 1):S191-4.

3 Kerr GS, Hallahan CW, Giordano J, Leavitt RY, Fauci AS, Rottem M. Takayasu arteritis. Ann Intern Med 1994;120:919-29.

4 Zhuang $\mathrm{H}$, Alavi A. 18-Fluorodeoxyglucose positron emission tomographic imaging in the detection and monitoring of infection and inflammation. Semin Nucl Med 2002;32:47-59

5 Matsunaga N, Hayashi K, Sakamoto I, Matsuoka Y, Ogawa Y, Honjo K, et al. Takayasu arteritis: MR manifestations and diagnosis of acute and chronic phase. J Magn Reson Imaging 1998;8:406-14.

6 Arend WP, Michel BA, Bloch DA, Hunder GG, Calabrese LH, Edworthy SM. The American College of Rheumatology 1990 criteria for the classification of Takayasu arteritis. Arthritis Rheum 1990;33:1129-34.

7 Marcos HB, Choyke PL. Magnetic resonance angiography of the kidney. Semin Nephrol 2000;20:450-5.

8 Tso E, Flamm SD, White RD, Schvartzman PR, Mascha E, Hoffman GS. Takayasu arteritis: utility and limitations of magnetic resonance imaging in diagnosis and treatment. Arthritis Rheum 2002;46:1634-42.

9 Blockmans D, Maes A, Stroobants S, Nuyts J, Bormans G, Knockaert D, et al. New arguments for a vasculitic nature of polymyal gia rheumatica using positron emission tomography. Rheumatology (Oxford) 1999;38:444-7.

10 Belhocine T, Blockmans D, Hustinx R, Vandevivere J, Mortelmans L. Imaging of large vessel vasculitis with (18)FDG PET: illusion or reality? A critical review of the literature data. Eur J Nucl Med Mol lmaging 2003;30:1305-13.

11 Meller J, Grabbe E, Becker W, Vosshenrich R. Value of F-18 FDG hybrid camera PET and MRI in early Takayasu aortitis. Eur Radiol 2003;13:400-5.

12 Meller J, Strutz F, Siefker U, Scheel A, Sahlmann CO, Lehmann K, et al. Early diagnosis and follow-up of aortitis with [(18)F]FDG PET and MRI. Eur J Nucl Med Mol Imaging 2003;30:730-6.

\section{Making Health Care Safer 2004}

21-22 October 2004

Royal College of Physicians, London

A two day conference for all professionals dedicated to providing safer health care for all.

Register now! Early booking discount available.

See website for details: www.quality.bmipg.com 